\title{
In vitro conservation of fruit trees by slow growth storage
}

\author{
A. Arbeloa, J.A. Marín, P. Andreu, E. García and P. Lorente \\ Estación Experimental de Aula Dei-CSIC \\ Avda. Montañana 1005 \\ 50059 Zaragoza.Spain \\ e-mail: arbeloa@eead.csic.es
}

Key words: Low temperature, darkness, nutrient limitation Prunus, Punica, Ficus, Cydonia, Pyrus, Malus, Eriobotrya, Crataegus.

\begin{abstract}
In vitro culture has been confirmed today as a useful tool that facilitates multiplication and conservation of genetic resources from vegetative propagated species. In vitro slow growth enables storage with lower labor costs. Low temperature, darkness and an adequate culture medium were considered when establishing cultures under slow growth conditions. The aim of this work is to develop a routine protocol to maintain cultures for a wide variety of fruit tree clones. We have conserved 138 fruit-tree clones belonging to 18 species from 8 genera: Prunus, Punica, Ficus, Cydonia, Pyrus, Malus, Eriobotrya and Crataegus. The cultures were maintained at $3-4^{\circ} \mathrm{C}$ both in darkness or continuous lighting in two different culture media for 7 or 12 months. Culture media were determinant in multiplication and length of recovery in the genera Crataegus and Eriobotrya while not in the rest. Continuous lighting was detrimental under these conditions for all clones. Conservation for 7 or 12 months did not affect survival rates although more shoots died. Only two clones out of 138, 'Cadaman' (Prunus persica $x$ P.davidiana) and one clone of Punica granatum, did not adapt to these slow growth conditions. The protocol presented here enabled the conservation for 12 months of $99 \%$ of the clones.
\end{abstract}

\section{INTRODUCTION}

Fruit tree germplasm is nowadays threatened by changes in land use and continuous renewal of cultivars. Germplasm conservation is thus a priority task. Fruit tree collections are normally held in orchards, but additional storage systems may be needed to increase security since field genebanks are open to loss risks.

In vitro conservation is a very useful tool for conservation of plants that require vegetative propagation. It offers a suitable alternative to the field collections and therefore has been widely used. Although this system presents great advantages as high multiplication rates, aseptic conditions or reduced space requirements, it is also an expensive technique, mainly due to high labor costs. For medium term storage the aim would be to reduce plant growth and plant manipulation, increasing the intervals between subcultures (Engelmann, 1991a). Growth reduction is generally achieved by culture medium modifications, or by environmental changes like temperature, photoperiod, culture vessel, oxygen level etc. (George, 1993). The most widely used change is temperature reduction, which can be combined with a decrease in light intensity or even darkness (Lambardi and Ozudogru, 2013). Modifications in culture medium might include dilution of mineral elements, reduction of sucrose concentration, changes in growth regulators balance or addition of osmotically active compounds.

Slow growth storage is routinely employed for a large number of species although its implementation still needs customizing to any new material. Working with 
different genotypes usually provides different results in terms of conservation (Engelmann, 1991b; Lambardi and Ozudogru, 2013). Moreover, it is not always possible to apply one single protocol for conserving genetically diverse material (Engelmann, 2011).

Slow growth has been applied for conservation in some fruit trees (Wilkins et al., 1988) but protocols are still needed for other fruit species or cultivars (Negri et al., 2000). An in vitro collection of fruit trees including 138 fruit tree clones belonging to 18 species from 8 genera was maintained as a duplicate to the field collections at the Aula Dei Experimental Station. In this work a medium term in vitro conservation protocol has been developed that is valid for a wide number of fruit tree cultivars.

\section{MATERIALS AND METHODS}

In this work 138 clones have been cultivated in vitro under slow growth conditions. The clones belong to 18 different species from 8 genera, being Prunus the most abundant with 125 different clones (Table 1).

\section{In vitro culture}

Plant material have been successfully cultured for several months, as previously described (Andreu and Marín, 2005), in a modified Murashige and Skoog (1962) MS medium with $0.4 \mathrm{mg} / \mathrm{L}$ thiamine- $\mathrm{HCl}, 5.0 \mathrm{mM}$ 6-benzylaminopurine (BAP), $0.5 \mathrm{mM}$ indole 3-butyric acid (IBA), 3\% sucrose, pH 5.7 and gelled with $0.7 \%$ Difco-Bacto agar. Culture chamber was maintained at $24^{\circ} \mathrm{C}$ and a 16-h photoperiod with cool-white fluorescent tubes $\left(35 \mu \mathrm{mol} \mathrm{m} \mathrm{s}^{-2}\right)$.

\section{Slow growth culture}

For slowing down the growth, the cultures were maintained at $3-4^{\circ} \mathrm{C}$ under different conditions. Both continuous lighting with white LEDs (3 watts, 110-150 lm), or continuous darkness were tested. Two modifications to the standard MS culture medium were applied: sucrose was reduced to $2 \%$ (MS20) and, in addition, macronutrients were reduced to half concentration (1/2MS20). And finally, two storage periods were tested: 7 or 12 months, without any subculture.

Twelve shoots per treatment and clone were kept for two weeks at $24^{\circ} \mathrm{C}$ and $16 \mathrm{~h}$ photoperiod in two jars with slow growth medium before they were moved to the low temperature chamber $\left(4^{\circ} \mathrm{C}\right)$ for conservation.

Plant condition was controlled every 4 weeks during storage attending necrosis, senescence, shoot brownish or yellowing, apical tip development or the presence of basal callus. At the end of each treatment multiplication rates (number of shoots/shoot), shoot elongation (elongated: doubles initial length), plant condition, and survival percentage were scored.

\section{Recovery}

Recovery of the material upon completion of the different treatments begins with subculturing the shoots on standard conditions: MS medium, 23-25 ${ }^{\circ} \mathrm{C}$ and 16 -hour photoperiod. After cold storage, three consecutive subcultures were performed on MS medium at monthly intervals and then the optimal growth of the material was evaluated. Recovery duration was sometimes longer and the number of subcultures and the time required to reach optimal growth were scored for every clone.

\section{RESULTS AND DISCUSSION}


This work shows that under slow growth conditions different fruit tree species and cultivars may be stored efficientely. We have achieved a survival rate of $98.6 \%$ under low temperature (136 out of 138 clones were recovered after 12 months without any subculturing). A general protocol, valid for 17 species belonging to 7 genera, has been established. However, one clone of Punica granatum as well as the hybrid Prunus persica $x$ P.davidiana did not survive in any of the two culture media tested. The death of these clones might indicate a low adaptation to certain culture conditions as low temperature, since both clones needed in our lab higher temperatures than other cultivars to grow in vitro. It has been previously stated that shoots of Punica granatum, Morus nigra, and Actinida chinensis only survived at $10^{\circ} \mathrm{C}$ or $25^{\circ} \mathrm{C}$, showing varying degrees of chilling sensitivity at $4^{\circ} \mathrm{C}$ (Wilkins et al., 1988). In general, previous works in woody fruit trees used a temperature between $1^{\circ} \mathrm{C}$ and a maximum of $12^{\circ} \mathrm{C}$ for slow growth conservation (Galzy and Compan, 1988; Lundergan and Janick, 1979). However, in most of the works, as well as in ours, a temperature between 2 and $5^{\circ} \mathrm{C}$ was used (Lambardi and Ozodugro, 2013).

We have stated here that when working with a new species or even a new clone, it must be experienced before to see its adaptation to cold, culture medium or other slow growth conditions.

\section{Darkness/light effect}

Maintaining the cultures under slow growth conditions with continuous illumination caused the rapid decay of all crops indicating that the presence of continuous light is unfavorable for the preservation of this material. After two and a half months under continuous light the treatment was suspended due to the bad aspect of the shoots with massive defoliation and the risk of loosing the plant material, being eliminated for the following experiments. Some photoperiod combinations provided good results in Prunus (Marino et al., 1985) or Malus (Hao and Deng, 2003), although most storage conditions were performed in darkness. In our work, continuous ligthing resulted not adequate to preserve any of the clones studied.

\section{Culture media effect}

Two culture media have been tested and both presented a very similar survival rate close to $98 \%$ of the clones after 7 months in slow growth conditions. It should be noted that within the genus Ficus the culture medium resulted decisive, since the cultivar 'Napolitana' only survived in 1/2MS20 medium.

Main differences between both culture media were found in multiplication rates, being higher in $1 / 2$ MS20 than in MS20. One third of the clones showed a higher multiplication rate in $1 / 2 \mathrm{MS} 20$ medium compared with the $10 \%$ of the clones with a higher rate in MS20 medium (Figure 1). More specifically, within the wide group of Prunus, $31.4 \%$ of the clones presented higher rates in $1 / 2 \mathrm{MS} 20$ compared with the $20 \%$ of the clones with a higher rate in MS20. Whithin Crataegus differences between media were most noticeable with an average multiplication rate of only 1.2 in MS20, whereas the rate increased up to 13 in $1 / 2 \mathrm{MS} 20$ medium (Figure 1).

As for the shoot elongation no significant differences between the two culture media were appreciated since most of the clones elongates either in both media or in none. Eriobotrya is the only genus where shoots showed elongation in MS20 and did not elongate in $1 / 2 \mathrm{MS} 20$.

The favorable effect of $1 / 2 \mathrm{MS} 20$ medium on multiplication rates and conservation could be related either to nutritive or osmotic effects of reducing sugar and mineral nutrients. Half macronutrientents concentration were applied to other fruit trees 
conservation as apple (Negri et al., 2000) or pear (Ahmed et al., 2010) and 20g/L of sucrose were used with some Prunus rootstocks (Marino et al., 1985), Pyrus and Malus (Hao and Deng, 2003; 2005) under slow growth storage. In our work, 1/2MS20 medium resulted determinant in some clones and appeared to be more favorable for the conservation of 136 clones during 12 months at low temperatures, since multiplication and elongation were higher and this had a great influence on recovery duration.

\section{Conservation period}

When conservation period varied from 7 to 12 months in $1 / 2 \mathrm{MS} 20$ medium at dark and $4^{\circ} \mathrm{C}$, survival rates were the same (near 99\%). However, decay in the state of some shoots was observed when time is lengthened. Although more shoots died in some clones when cultures were prolonged for a period of 12 months, this fact did not imply the loss of clone viability. Multiplication rate even increased in some other clones, like those of Crataegus or 'Djerba' apple, when slow growth storage was extended from 7 to 12 months. Most plant cultures were preserved for 9 to 36 months in slow growth conditions (Withers et al., 1990; Withers, 1992) and specifically for fruit tree species a conservation time of 9 months were common, with a maximum of 24 months for apple rootstocks (Wilkins et al., 1988).

\section{Recovery}

Recovery period after storage of plants under slow growth conditions must be as short as possible to return cultures to in vitro optimal growth to be ready for their use in further experiments with minimum effort. In general, we observed that the number of subcultures necessary for recovery of cultures to active growth was 3 subcultures (3 months) when conserved in optimal $1 / 2 \mathrm{MS} 20$ medium at darkness and $4^{\circ} \mathrm{C}$ conditions during 12 months (Figure 2). However, the results varied with genotype and culture media, thus within Prunus, recovery after 3 subcultures took place in only $49.2 \%$ of the clones in MS20 medium compared to $81 \%$ of them in $1 / 2 \mathrm{MS} 20$ medium, while Eriobotrya and Ficus recovered faster in MS20 (Figure 3). According to Lambardi and Ozudogru (2013) when plants are transferred back to standard culture medium, they should be able to resume quickly their initial growth rate in 1-2 subcultures, which is close to the results achieved in our cultures.

The present protocol, based on the conservation under darkness, at $4^{\circ} \mathrm{C}$, and $1 / 2 \mathrm{MS} 20$ culture medium, during 12 months without subculturing, enabled the storage of $98.6 \%$ of the clones studied and the recovery of the cultures in 3 subcultures.

\section{ACKNOWLEDGMENTS}

This work was partly funded by the INIA-FEDER RTA2010-00053-C03 project and by Group A-43 Excellence grant by the Government of Aragon.

\section{Literature Cited}

Ahmed, M., Anjum, M. A., Sahah, A. H., S. and Hamid, A. 2010. In vitro preservation of Pyrus germplasm with minimal growth using different temperature regimes. Pak. Journal Botany, 42(3): 1639-1650.

Andreu, P.and Marín, JA. 2005. In vitro culture establishment and multiplication of the Prunus rootstock 'Adesoto 101' ( $P$. insititia L.) as affected by the type of propagation of the donor plant and by the culture medium composition. Scientia Horticulturae 106: 258-267 
Engelmann, F. 1991a. In vitro conservation of horticultural species. Acta Horticulturae 298: 327-334.

Engelmann, F. 1991b. In vitro conservation of tropical plant germplasm - a review. Euphytica 57: 227-243.

Engelmann, F. 2011. Use of biotechnologies for the conservation of plant biodiversity. In Vitro Cell Dev. Biology- Plant 47: 5-16

Galzy, R. and Compan, D. 1988. Growth and nutrition of grapevine during in vitro long-term storage. Plant Cell, Tissue and Organ Culture 13:229-237.

George, E.F. 1993. Plant propagation by tissue culture. Part 1. The technology. Ed: Exegenetics Limited, England. pp: 163-182.

Hao, Y-J. and Deng, X-X. 2003. Genetically stable regeneration of apple plants from slow growth. Plant Cell, Tissue and Organ Culture 72: 253-260.

Hao, Y-J. and Deng, X-X. 2005. Stable maintenance and expression of a foreign gene in transgenic pear shoots retrieved from in vitro conservation. Journal of Plant Physiology 162: 237-243.

Lambardi, M. and Ozudogru, E.A. 2013. Advances in the safe storage of micropropagated woody plants at low temperature. Acta Horticulturae 988: 2942.

Lundergan, C. and Janick, J. 1979. Low temperature storage of in vitro apple shoots. Hortscience, 14:514.

Marino, G., Rosati, P. and Sagrati, F. 1985. Storage of in vitro cultures of Prunus rootstocks. Plant Cell, Tissue and Organ Culture, 5:73-78.

Murashige, T. and Skoog, F. 1962. A revised medium for rapid growth and bioassays with tobacco tissues cultures. Physiologia Plantarum, 15:473-479.

Negri, V., Tosti N. and Standardi A. 2000. Slow-growth storage of single node shoots of apple genotypes. Plant Cell, Tissue and Organ Culture, 62: 159-162.Withers, L.A., Wheelans, S.K. and Williams, J.T. 1990. In vitro conservation of crop germplasm and the IBPGR databases. Euphytica 45: 9-22.

Withers, L.A., Wheelans S.K. and Williams J.T. 1990. In vitro conservation of crop germplasm and the IBPGR databases. Euphytica 45: 9-22

Withers, L. A. 1992. In Vitro conservation. In: F. A. Hammerschlag \& R. E. Litz (Eds.). Biotechnology of Perennial Fruit Crops. Wallingford: CAB International.

Wilkins, C.P., Newbury, H.J. and Dodds, J.H. 1988. Tissue culture conservation of fruit trees. FAO/IBPGR Plant Gen. Res. Newsl. 73/74: 9-20. 


\section{Tables}

Table 1. Number of clones from each species cultivated in vitro under slow growth conditions

\begin{tabular}{|l|l|c|}
\hline Genus & Species & Number of clones \\
\hline Prunus & Prunus cerasifera & 10 \\
\hline & Prunus cerasifera $x$ P. munsoniana & 4 \\
\hline & Prunus cerasifera x P.armeniaca & 77 \\
\hline & Prunus domestica & 15 \\
\hline & Prunus insititia & 1 \\
\hline & Prunus cerasus & 2 \\
\hline & Prunus avium x pseudocerasus & 1 \\
\hline & Prunus persica & 1 \\
\hline & Prunus amygdalus $x$ P.persica & 6 \\
\hline & Prunus persica. $x$ P.davidiana & 2 \\
\hline Malus & Prunus spinosa & 6 \\
\hline Pyrus & Malus domestica & 2 \\
\hline Ficus & Pyrus communis & 1 \\
\hline Punica & Ficus carica & 2 \\
\hline Cydonia & Punica granatum & 1 \\
\hline Eriobotrya & Cydonia oblonga & 2 \\
\hline Crataegus & Eriobotrya japonica & 2 \\
\hline TOTAL & Crataegus monogyna & 3 \\
\hline
\end{tabular}

\section{Figures}

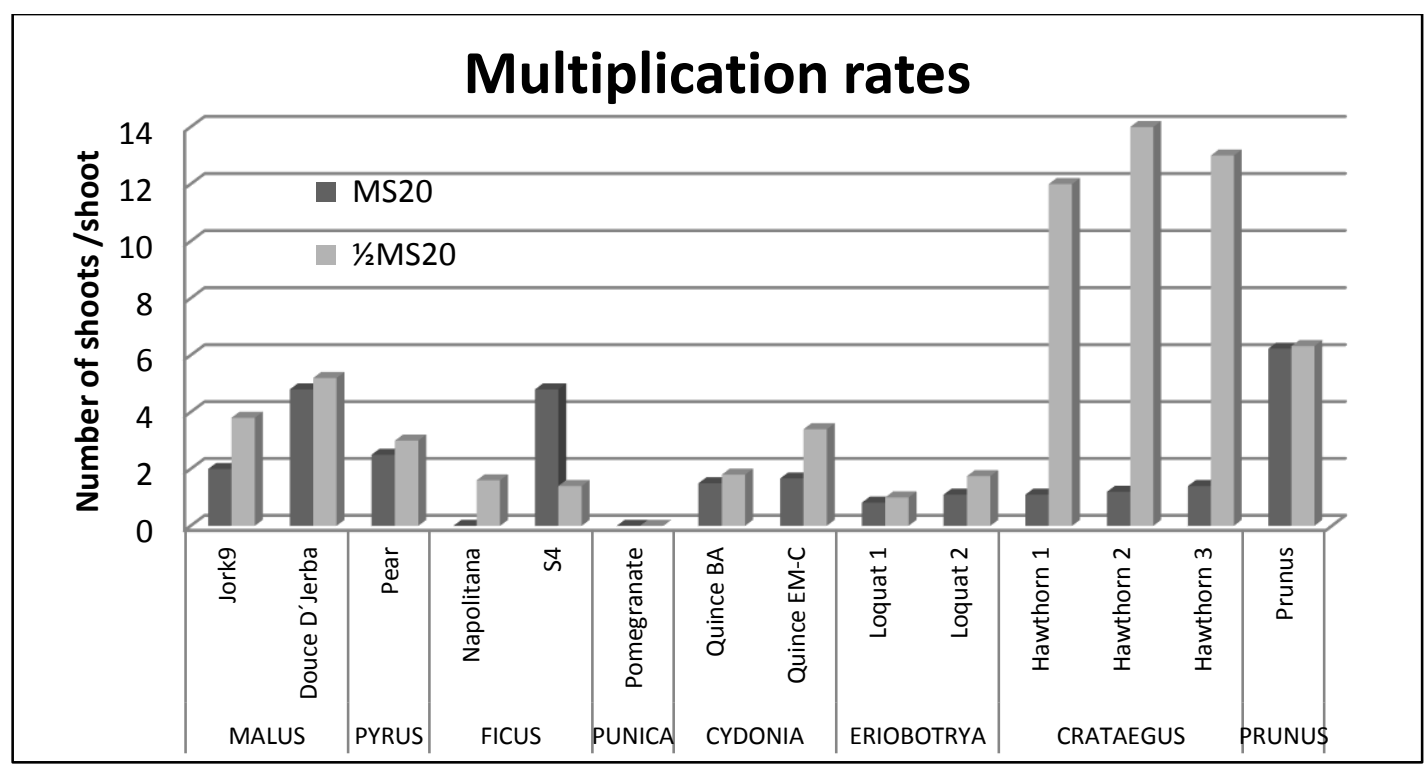

Figure 1. Multiplication rates of different species in two culture media: MS20 and $1 / 2$ MS20 after seven months under slow growth conditions. 

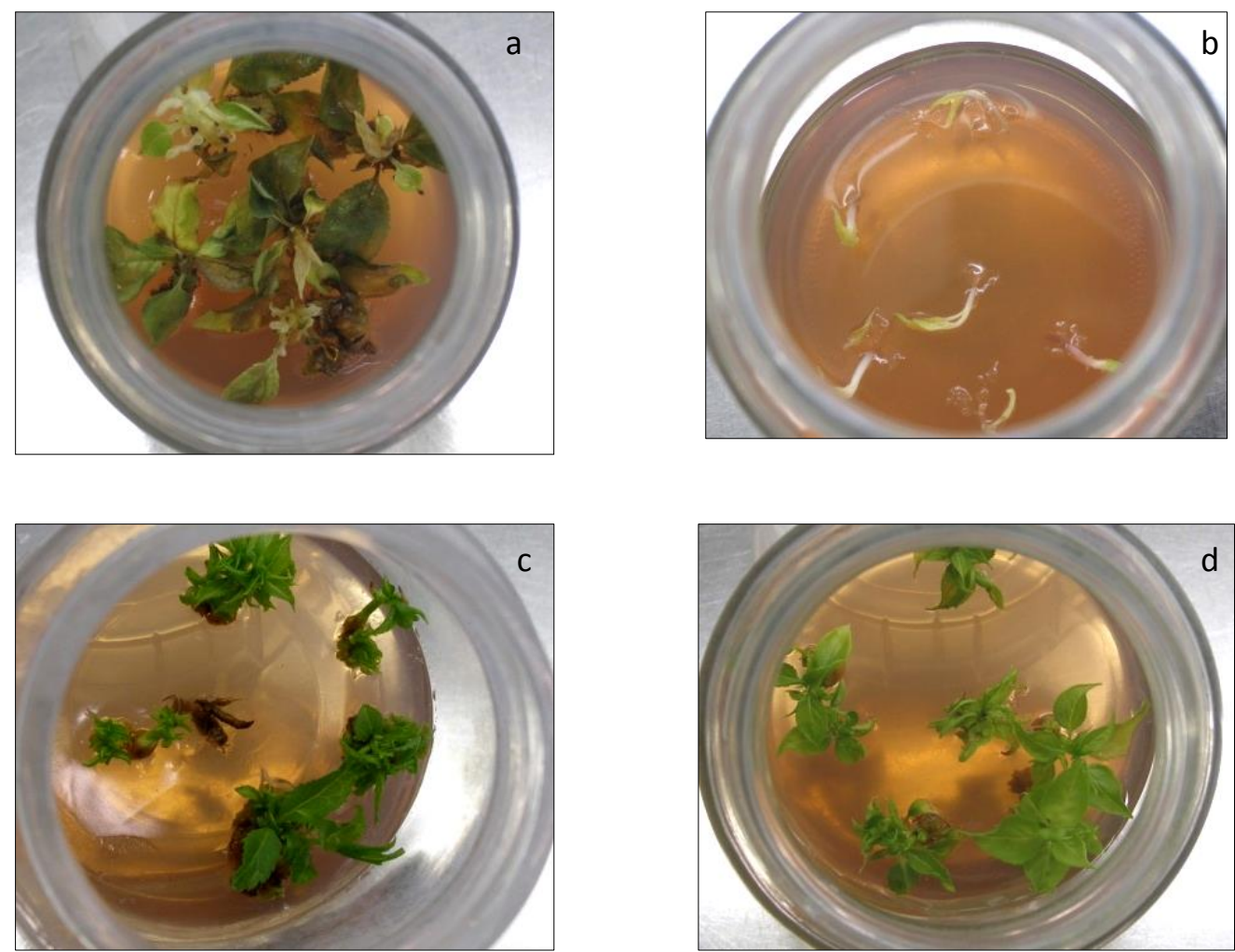

Figure 2. Developmental stages of the recovery of the hybrid Prunus cerasifera $x$ P.armeniaca after 12 months in slow growth conditions in $1 / 2$ MS20 medium (a), first subculture (b), second subculture (c) and third subculture (d)

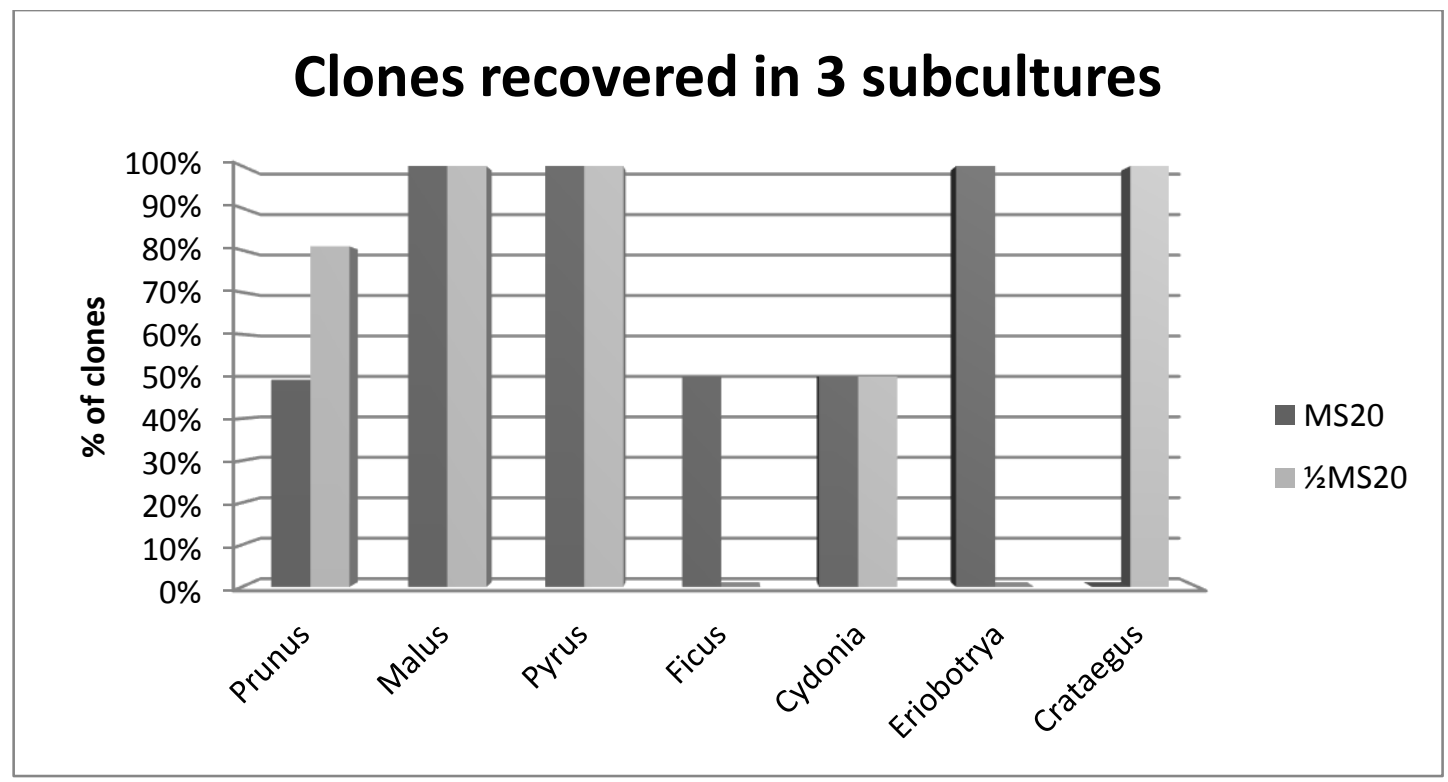

Figure 3. Effect of culture media: MS20 or $1 / 2 \mathrm{MS} 20$ in recovery time for different fruit tree genera. 